\title{
Assessment of Different Types of Strabismus Among Pediatric Patients in a Tertiary Hospital in Jeddah
}

Ahmed S. Qanat ${ }^{1}$, Abdullah Alsuheili ${ }^{2}$, Abdulkarim M. Alzahrani ${ }^{2}$, Abdulrahman A. Faydhi ${ }^{2}$, Abdulhadi Albadri $^{2}$, Nizar Alhibshi ${ }^{2}$

1. Research, King Abdulaziz University Hospital, Jeddah, SAU 2. Ophthalmology, King Abdulaziz University Hospital, Jeddah, SAU

Corresponding author: Ahmed S. Qanat, ahmedsalahqanat@gmail.com

\section{Abstract \\ Background}

Strabismus, also known as squint, is an ocular disorder in which the eyes do not align properly with each other when looking at an object. The estimated global prevalence of strabismus among children is between $1.3 \%$ and $5.7 \%$. This study aimed to assess the various types of strabismus among pediatric patients in Jeddah, in the western region of Saudi Arabia.

\section{Methods}

The medical records of 281 patients with strabismus aged $\leqslant 18$ years, who presented to the pediatric ophthalmology clinic in King Abdulaziz University Hospital between 2010 and 2019, were retrospectively reviewed. Data were analyzed using the Statistical Package for Social Sciences (SPSS; IBM Corp., Armonk, NY, USA). A p-value of 0.05 or less was considered statistically significant.

\section{Results}

Out of the 281 patients, 141 were (50.2\%) female. The average age of the patients was $9.50 \pm 4.24$ years. The most common type of strabismus was esotropia (177 [63\%] patients), followed by exotropia (96 [34.2\%] patients), hypertropia (10 [3.6\%] patients), and dissociated vertical deviation (four [1.4\%] patients). Twohundred thirty-one (82.8\%) patients had bilateral strabismus. A total of 178 patients (63.3\%) had no associated conditions with strabismus, whereas 103 (36.7\%) had an associated condition. A significant relationship was observed between esotropia and prematurity $(\mathrm{p}=0.024)$.

\section{Conclusion}

Esotropia was the most common type of strabismus among the patients, followed by exotropia. The results of this study showed that males were equally affected as females. We also found a significant relationship between esotropia and prematurity. Implementation of a compulsory nationwide pediatric ophthalmic

Review began 11/17/2020 Review ended 12/03/2020 Published 12/08/2020

\section{(c) Copyright 2020}

Qanat et al. This is an open access article distributed under the terms of the Creative Commons Attribution License CC-BY 4.0., which permits unrestricted use, distribution, and reproduction in any medium, provided the original author and source are credited. screening program for children aged one, three, and five years is recommended to enable timely diagnosis of strabismus and any other refractive errors.

\footnotetext{
Categories: Ophthalmology

Keywords: strabismus, pediatric, esotropia, exotropia, jeddah, saudi arabia, hypertropia, dissociated vertical deviation
}

\section{Introduction}

Strabismus, commonly referred to as squint, cross-eyes, walleyes, wandering eyes, or deviating eyes [1], is an ocular condition characterized by misalignment of the eyes when viewing an object; this misalignment may be continuous or intermittent [2]. Based on the direction of the deviated eye, strabismus may be classified as esotropia (inward deviation), exotropia (outward deviation), hypertropia (upward deviation), or hypotropia (downward deviation) [3]. An infant with strabismus may stop using the impaired eye, resulting in a visual impairment termed as amblyopia, which may become permanent if not treated early. Since strabismus may also inhibit the development of binocular vision, early intervention is vital to ensure optimal visual function [1,4]. Due to the visual problems associated with it, strabismus may leave a negative psychosocial impact on the child. In addition, children with strabismus have a reduced vision-related quality of life in comparison to children with normal vision. Moreover, the parents of these children also experience a reduced quality of life $[5,6]$. Strabismus is associated with various risk factors such as low gestational age, reduced birth weight, family history of squint [7], neuromuscular disorders, maternal smoking during pregnancy, cataracts, head injuries, tumors of the brain or eyes, and systemic conditions that have ocular manifestations [1]. 
Strabismus is not a rare condition, and the global prevalence among children is about $1.3 \%-5.7 \%$ [8]. In a study conducted in Saudi Arabia in 2015 to assess the pattern of eye diseases among pediatric patients aged one to 14 years, the estimated prevalence of strabismus was reported as $11.8 \%$ [9]. In a retrospective, population-based, cohort study conducted in Olmsted County, Minnesota, USA, 627 new cases of childhood squint were recognized during the 10-year study period, which included 380 (60.1\%) cases of esotropia, 205 (32.7\%) of exotropia, and 42 (6.7\%) of hypertropia [10]. In contrast, a Japanese study has shown different prevalence statistics, in which exotropia was predominant [11]. Additionally, a population-based study conducted in Palestine, Gaza Strip, in 2014, indicated esotropia as the most prevalent type of squint [12].

Regarding studies conducted in Saudi Arabia, a study on 385 patients who presented to the pediatric ophthalmology clinic in Jazan, Saudi Arabia, between October 2014 and October 2015 revealed that strabismus (36.9\%) was the most common eye disorder observed [13]. A recent cross-sectional study conducted on 156 randomly selected participants in Arar, northern Saudi Arabia, showed that 14.7\% of them were strabismic; among these, $78.3 \%$ had esotropia, and $17.4 \%$ had exotropia. The study further demonstrated that strabismus has a significant relationship with consanguinity [14]. In a study conducted in the middle region of Saudi Arabia, which included 4,886 patients with strabismus who had undergone surgery in Riyadh, esotropia was the most common type of strabismus (69.3\%), whereas exotropia was less prevalent (26.9\%) [15]. A clinic-based study performed in the eastern region of Saudi Arabia in 2015, which included 1,350 children, revealed that $38 \%$ of the participants had strabismus [16].

A search of the available literature revealed limited local data regarding the prevalence of strabismus among children in Jeddah, which is in the western region of Saudi Arabia. To fill this gap, we conducted this study to assess the various types of strabismus among pediatric patients in Jeddah.

\section{Materials And Methods}

This was a retrospective medical record review study. The study was approved by the institutional review board of King Abdulaziz University Hospital (KAUH) (Reference Number 304-20). No names or identification numbers were recorded to ensure patient confidentiality. All patients provided informed consent to participate in this study. We recruited patients aged 18 years or less who were seen at the ophthalmology clinic of our hospital and were diagnosed with strabismus. This study was conducted between June and September 2020 .

A total of 471 medical records from 2010 to 2019 were reviewed; among these, 281 patients met our inclusion criteria. Patients with missing data were excluded from the study. Data were extracted from the electronic-based medical record system of KAUH (Phoenix). The extracted data included demographic data, age at the time of diagnosis, type of strabismus, test/s performed, laterality of the affected eye, and other associated conditions. The patients were categorized into three groups according to their age at the time of this study: one to six years, seven to 12 years, and 13 to 18 years.

All data were entered into Microsoft Excel version 2010. The Statistical Package for Social Sciences (SPSS) version 21 (IBM Corp., Armonk, NY, USA) was used for data analysis. Categorical variables were expressed as frequencies and percentages, whereas continuous data were presented as means \pm standard deviations. The chi-square test was used to calculate the correlations between variables. A p-value of 0.05 or less was considered statistically significant.

\section{Results}

A total of 281 medical records of pediatric patients with strabismus were reviewed; 141 (50.2\%) patients were female and 140 (49.8\%) were male. Out of the 281 patients, 207 (73.7\%) were Saudi nationals, whereas 74 (26.3\%) were not. The mean age of the patients was $9.50 \pm 4.24$ years and the mean age at the time of their diagnosis was $5.19 \pm 3.53$ years. The seven to 12 years age group had the highest frequency with $140(49.8 \%)$ patients, followed by the one to six years group with 75 (26.7\%) and the 13 to 18 group with 66 (23.5\%) patients. The most common type of strabismus was esotropia (177 [63\%] patients), followed by exotropia (96 [34.2\%] patients) (Table 1). 


\section{Cureus}

\begin{tabular}{|c|c|c|c|c|c|}
\hline \multirow{2}{*}{ Variables } & & \multicolumn{4}{|l|}{ Type of strabismus } \\
\hline & & Esotropia No. (\%) & Exotropia No. (\%) & Hypertropia No. (\%) & Dissociated vertical deviation No. (\%) \\
\hline \multirow{3}{*}{ Age groups } & $1-6$ years & $56(31.6)$ & $16(16.7)$ & $3(30)$ & $1(25)$ \\
\hline & $7-12$ years & 91 (51.4) & $47(49)$ & $3(30)$ & $1(25)$ \\
\hline & 13-18 years & $30(16.9)$ & $33(34.4)$ & $4(40)$ & $2(50)$ \\
\hline \multirow{2}{*}{ Gender } & Male & $89(50.3)$ & $48(50)$ & $5(50)$ & $3(75)$ \\
\hline & Female & $88(49.7)$ & $48(50)$ & $5(50)$ & $1(25)$ \\
\hline \multirow{2}{*}{ Nationality } & Saudi & 135 (76.3) & $64(66.7)$ & $10(100)$ & $2(50)$ \\
\hline & Non-Saudi & $42(23.7)$ & $32(33.3)$ & $0(0)$ & $2(50)$ \\
\hline \multirow{2}{*}{ Affected eye } & Unilateral & $23(13.1)$ & $17(17.9)$ & $8(80)$ & $0(0)$ \\
\hline & Bilateral & $153(86.9)$ & $78(82.1)$ & $2(20)$ & $4(100)$ \\
\hline Total (\%) & & 177 (63) & $96(34.2)$ & $10(3.6)$ & $4(1.4)$ \\
\hline
\end{tabular}

TABLE 1: Type of squint and demographics of the sample $(n=281)$.

Among the 281 patients, 231 (82.8\%) showed bilateral involvement, whereas 48 (17.1\%) showed unilateral involvement. The most common ophthalmic tests employed in our study were the cover test, followed by cycloplegic refraction, Hirschberg test, and Krimsky test, which were performed in 92 (32.7\%), 63 (22.4\%), 44 (15.7\%), and 27 (9.6\%) patients, respectively. Table 2 shows that 178 (63.3\%) patients had no associated conditions with strabismus, whereas 103 (36.7\%) had associated conditions. The most common condition associated with squint was developmental delay. 


\section{Cureus}

\begin{tabular}{|c|c|c|c|}
\hline \multicolumn{2}{|l|}{ Associated conditions } & Frequency & Percentage \% \\
\hline \multirow{4}{*}{ Psychiatric/developmental disorders: } & Developmental delay & 29 & 10.3 \\
\hline & Autism & 2 & 0.7 \\
\hline & ADHD & 2 & 0.7 \\
\hline & Mental retardation & 1 & 0.4 \\
\hline \multirow{4}{*}{ Neurological disorders: } & Epilepsy & 16 & 5.7 \\
\hline & Hydrocephalus & 16 & 5.7 \\
\hline & Cerebral palsy & 12 & 4.3 \\
\hline & Brain tumor & 4 & 1.4 \\
\hline \multirow{4}{*}{ Ophthalmic disorders: } & Cataract & 6 & 2.1 \\
\hline & Glaucoma & 3 & 1.1 \\
\hline & Optic atrophy & 2 & 0.7 \\
\hline & Duane syndrome & 1 & 0.4 \\
\hline \multirow{3}{*}{ Infectious disorders: } & Hepatitis C & 7 & 2.5 \\
\hline & Congenital toxoplasmosis & 1 & 0.4 \\
\hline & Infectious mononucleosis & 1 & 0.4 \\
\hline \multirow{2}{*}{ Endocrine disorders: } & Hypothyroidism & 14 & 5 \\
\hline & Diabetes Mellitus & 3 & 1.1 \\
\hline \multirow{7}{*}{ Syndromes: } & Down syndrome & 7 & 2.5 \\
\hline & Alagille syndrome & 1 & 0.4 \\
\hline & Dandy-walker syndrome & 1 & 0.4 \\
\hline & William's syndrome & 1 & 0.4 \\
\hline & Kabuki syndrome & 1 & 0.4 \\
\hline & ROOHAD syndrome & 1 & 0.4 \\
\hline & Freeman-Sheldon syndrome & 1 & 0.4 \\
\hline \multirow{6}{*}{ Other: } & Prematurity & 23 & 8.2 \\
\hline & Craniofacial anomalies & 11 & 3.9 \\
\hline & Anemia & 9 & 3.2 \\
\hline & Asthma & 3 & 1.1 \\
\hline & Low birth weight & 1 & 0.4 \\
\hline & Chiari malformation & 1 & 0.4 \\
\hline Non & & 178 & 63.3 \\
\hline
\end{tabular}

TABLE 2: Frequency and percentage of associated conditions.

ADHD: Attention deficit hyperactivity disorder; ROOHAD: rapid-onset obesity with hypothalamic dysregulation, hypoventilation, and autonomic dysregulation.

No significant relationship was observed between the affected eye (unilateral or bilateral) and exotropia or dissociated vertical deviation; however, a significant association was observed with esotropia and hypertropia ( $p=0.026$ and $p=0.000$, respectively). No significant relationship was observed between developmental delay and esotropia or exotropia ( $\mathrm{p}=0.925$ and $\mathrm{p}=1.00$, respectively). However, there was a 
significant association between prematurity and esotropia and exotropia $(\mathrm{p}=0.024$ and $\mathrm{p}=0.037$, respectively).

Esotropia was the most common condition in the one to six (56 [74.7\%] patients) and sven to 12 (91 [65\%] patients) age groups. Exotropia was most common in the 13 to 18 years age group (33 [50\%] patients). We observed a significant relationship between esotropia, exotropia, and all age groups $(\mathrm{p}=0.001$ for esotropia; $\mathrm{p}=0.002$ for exotropia). No significant association was observed between sex and any type of strabismus.

\section{Discussion}

In this study, we assessed the various types of strabismus among pediatric patients in Jeddah; esotropia was observed to be the most predominant type of strabismus among these patients. This finding is in line with those of other studies conducted in Arar city and Riyadh, Saudi Arabia [14,15]. Similar results were reported in a Palestinian study [12], the study by Medghalchi [17], Mvogo et al. [18], and the study conducted in Olmsted County, USA [10]. In contrast, exotropia is reportedly more common than esotropia in East Asia. Two Chinese studies reported exotropia to be the most common type of strabismus, with a percentage of $80.94 \%$ reported in one study and $94.4 \%$ in the other [19,20]. Additionally, exotropia is reportedly the most common type of strabismus in Japan [11]. Furthermore, other studies conducted in Iran have indicated that exotropia is the most common type of strabismus in the country $[21,22]$.

In contrast to the study conducted in Arar, in which bilateral involvement of the eyes was not common, most of the patients in our study had bilateral strabismus [14]. This disparity may be attributed to the differences in the study designs. The Arar study was a cross-sectional population-based study; the questionnaires used in the study were completed by either the mothers of the children or the adolescents themselves. We believe that this may have made the study vulnerable to recall bias, as the participants (or their mothers) may not have noticed that the squint was bilateral. In our study, we retrieved the data, which was documented by an ophthalmologist after ophthalmic examination, from the hospital's electronic record system.

Furthermore, we did not observe any significant association between sex and any type of strabismus in our study. A global meta-analysis, in which 56 articles were reviewed, has also shown no association between sex and the prevalence of squint [23]. We believe that this lack of association between sex and strabismus in previous studies has been a consequence of the approximate prevalence of strabismus between males and females.

The results of our study showed an association between prematurity and esotropia, which is supported by that of a study conducted in Germany [7], and that of a study by Gursoy et al. [24]. A study conducted in Baltimore, Maryland, USA, has suggested that prematurity is independently related to elevated risk for both esotropia and exotropia. However, with regard to strabismus and prematurity, it may be difficult to compare the findings of our study with those of previous studies due to the differences in sampling methods, diagnostic methods, and definitions of prematurity and squint [25]. Moreover, a prospective study has shown that the mechanism behind the relationship between prematurity and strabismus is unclear [26]. However, another study has suggested that retinopathy of prematurity during early gestational age is a predisposing factor that may lead to strabismus [27].

Although developmental delay was the most common condition associated with strabismus in our study, the association was not statistically significant. This finding was in contrast with that of previous studies $[28,29]$, which have indicated that strabismus is a common disorder significantly associated with developmental delay in children. This variation in the results may have been due to selection bias, the differences in sampling methods, and the different study settings. KAUH is a government tertiary hospital; therefore, most of the patients are from different ethnic groups with varied socio-economic backgrounds.

Our study revealed a significant relationship between esotropia, exotropia, and all pediatric age groups. This finding differs from that of a study conducted in Jazan, Saudi Arabia, in which the 0 to six years age group had the largest number of strabismus patients [13]. Moreover, according to a retrospective study performed in Sweden, the greatest prevalence of strabismus was observed in children aged four years old, followed by a declining trend. This was achieved through an ophthalmic screening program that begins at the age of four [30].

However, due to the lack of visual screening programs, different ethnic backgrounds of the residents, and the shortage of research on populations within the same age groups in Saudi Arabia, the findings of the abovementioned study may be complicated and difficult to elaborate in the current study.

Compared to the majority of previous studies, which studied strabismus in all age groups, the findings of our study add to a growing body of literature on the most common type of strabismus among the pediatric population. However, there are some limitations to this study. First, there were some difficulties in finding articles on similar topics and with similar methodologies in the available literature. Second, the documentation of visual acuity and family history in the hospital system was not sufficiently detailed. Lastly, 
there is a possibility of selection bias in the study due to its retrospective nature.

\section{Conclusions}

In this retrospective study, no significant association was observed between sex and prevalence of strabismus. Further, esotropia was the most common type of strabismus, followed by exotropia. Moreover, a significant association was observed between esotropia and prematurity.

Early screening and detection of strabismus is crucial for the proper management of strabismus and strabismic amblyopia. We recommend that a compulsory nationwide pediatric ophthalmic screening program for children aged one, three, and five years be carried out to enable timely diagnosis of strabismus and other refractive errors. Parent education may be promoted through the distribution of pamphlets on amblyopia and strabismus in clinics, hospitals, and medical centers. Periodic educational programs on pediatric visual problems and the importance of early detection and management, including screening when possible, should be conducted at different locations, including schools, shopping malls, and social networks. Cycloplegic refraction should be mandatory during early childhood for patients with strabismus to assess their total amount of actual refractive errors and to help clinicians determine the best treatment method for strabismus. Further studies are needed to clarify and understand the heritability of strabismus.

\section{Additional Information \\ Disclosures}

Human subjects: Consent was obtained by all participants in this study. The Biomedical Ethics Research Committee issued approval 304-20. The study was approved by the institutional review board of King Abdulaziz University Hospital (KAUH) (Reference Number 304-20). Animal subjects: All authors have confirmed that this study did not involve animal subjects or tissue. Conflicts of interest: In compliance with the ICMJE uniform disclosure form, all authors declare the following: Payment/services info: All authors have declared that no financial support was received from any organization for the submitted work. Financial relationships: All authors have declared that they have no financial relationships at present or within the previous three years with any organizations that might have an interest in the submitted work. Other relationships: All authors have declared that there are no other relationships or activities that could appear to have influenced the submitted work.

\section{Acknowledgements}

This study was part of a summer school 2020 research project, which was conducted under the supervision of Road of Change team, an institute for teaching research. The authors would like to thank the team for supporting this paper. No financial support was needed for this paper. We also would like to acknowledge Abdulkarim Jawhari and Maha Safhi for supporting this paper, and Mohammed Al-shmrani for reviewing the statistical analyses. We also would like to thank Editage (www.editage.com) for English language editing.

\section{References}

1. Shah J, Patel S: Strabismus:-symptoms, pathophysiology, management \& precautions . IJSR. 2015, 4:1510-4.

2. Gunton KB, Wasserman BN, DeBenedictis C: Strabismus. Prim Care. 2015, 42:393-407. 10.1016/j.pop.2015.05.006

3. Strabismus - American Association for Pediatric Ophthalmology and Strabismus . (2018). Accessed: June 12 , 2020: https://aapos.org/glossary/strabismus.

4. Pediatric Ophthalmology/Strabismus Summary Benchmarks - 2019. (2019). Accessed: June 13, 2020: https://www.aao.org/summary-benchmark-detail/pediatric-ophthalmology-strabismus-summary-benchma.

5. Hatt SR, Leske DA, Castañeda YS, et al.: Association of strabismus with functional vision and eye-related quality of life in children. JAMA Ophthalmol. 2020, 138:528-35. 10.1001/jamaophthalmol.2020.0539

6. Kothari M, Balankhe S, Gawade R, Toshnival S: Comparison of psychosocial and emotional consequences of childhood strabismus on the families from rural and urban India. Indian J Ophthalmol. 2009, 57:285-8. 10.4103/0301-4738.53053

7. Fieß A, Kölb-Keerl R, Schuster AK, Knuf M, Kirchhof B, Muether PS, Bauer J: Prevalence and associated factors of strabismus in former preterm and full-term infants between 4 and 10 years of age. BMC Ophthalmol. 2017, 17:228. 10.1186/s12886-017-0605-1

8. Group M-ePEDS: Prevalence of amblyopia and strabismus in African American and Hispanic children ages 6 to 72 months: the Multi-ethnic Pediatric Eye Disease Study. Ophthalmology. 2008, 115:1229-36.

9. Danish EY, Shaikh RM, Hadrawi MT: Pattern of pediatric eye diseases at King Fahad Armed Forces Hospital, Jeddah. PJHMS. 2015, 9:945-7.

10. Mohney BG: Common forms of childhood strabismus in an incidence cohort . Am J Ophthalmol. 2007, 144:465-7. 10.1016/j.ajo.2007.06.011

11. Matsuo T, Matsuo C: The prevalence of strabismus and amblyopia in Japanese elementary school children . Ophthalmic Epidemiol. 2005, 12:31-6. 10.1080/09286580490907805

12. Amer A: Relative prevalence of various types of strabismus in patients attending NGO's medical centers in Gaza Strip. Sci J Pub Health. 2015, 3:1-5. 10.11648/j.sjph.s.2015030101.11

13. Darraj A, Barakat W, Kenani M, et al.: Common eye diseases in children in Saudi Arabia (Jazan) . Ther Adv Ophthalmol. 2016, 8:39055. 10.4137/OED.S39055

14. Alenezi HM, Alruwaili AS, Abo El-Fetoh NM, et al.: Squint in children and adolescents, Arar, Northern Saudi 
Arabia. EJHM. 2018, 70:298-302. 10.12816/0043093

15. Curtis TH, McClatchey M, Wheeler DT: Epidemiology of surgical strabismus in Saudi Arabia. Ophthalmic Epidemiol. 2010, 17:307-14. 10.3109/09286586.2010.508351

16. Al-Tamimi ER, Shakeel A, Yassin SA, Ali SI, Khan UA: A clinic-based study of refractive errors, strabismus, and amblyopia in pediatric age-group. J Family Community Med. 2015, 22:158-62. 10.4103/22308229.163031

17. Medghalchi A: A study on prevalence of horizontal strabismus in patients under 14 years . J Guilan Uni Med Sci. 2003, 12:80-85.

18. Mvogo CE, Ellong A, Bella-Hiag AL, Luma-Namme H: Hereditary factors in strabismus (Article in French) . Santé. 2001, 11:237-9.

19. Chen X, Fu Z, Yu J, et al.: Prevalence of amblyopia and strabismus in Eastern China: results from screening of preschool children aged 36-72 months. Br J Ophthalmol. 2016, 100:515-9. 10.1136/bjophthalmol-2015306999

20. Fu J, Li SM, Liu LR, et al.: Prevalence of amblyopia and strabismus in a population of 7th-grade junior high school students in Central China: the Anyang Childhood Eye Study (ACES). Ophthalmic Epidemiol. 2014, 21:197-203. 10.3109/09286586.2014.904371

21. Yekta A, Fotouhi A, Hashemi H, et al.: The prevalence of anisometropia, amblyopia and strabismus in schoolchildren of Shiraz, Iran. Strabismus. 2010, 18:104-10. 10.3109/09273972.2010.502957

22. Yekta, AA, Hashemi H, Aziz A, Rezvan F, Moghaddam HO, Derakhshan, A: The prevalence of amblyopia and strabismus among school children in Northeastern Iran, 2011. Iran J Ophthalmol. 2012, 24:3-10.

23. Hashemi H, Pakzad R, Heydarian S, et al.: Global and regional prevalence of strabismus: a comprehensive systematic review and meta-analysis. Strabismus. 2019, 27:54-65. 10.1080/09273972.2019.1604773

24. Gursoy H, Basmak H, Bilgin B, Erol N, Colak E: The effects of mild-to-severe retinopathy of prematurity on the development of refractive errors and strabismus. Strabismus. 2014, 22:68-73. 10.3109/09273972.2014.904899

25. Cotter SA, Varma R, Tarczy-Hornoch K, et al.: Risk factors associated with childhood strabismus: the multiethnic pediatric eye disease and Baltimore pediatric eye disease studies. Ophthalmology. 2011, 118:2251-61. 10.1016/j.ophtha.2011.06.032

26. Pennefather PM, Clarke MP, Strong NP, Cottrell DG, Dutton J, Tin W: Risk factors for strabismus in children born before 32 weeks' gestation. Br J Ophthalmol. 1999, 83:514-8. 10.1136/bjo.83.5.514

27. VanderVeen DK, Coats DK, Dobson V, et al.: Prevalence and course of strabismus in the first year of life for infants with prethreshold retinopathy of prematurity: findings from the Early Treatment for Retinopathy of Prematurity study. Arch Ophthalmol. 2006, 124:766-73. 10.1001/archopht.124.6.766

28. Sandfeld Nielsen L, Jensen H, Skov L: Risk factors of ophthalmic disorders in children with developmental delay. Acta Ophthalmol. 2008, 86:877-81. 10.1111/j.1755-3768.2007.01158.x

29. Sandfeld Nielsen L, Skov L, Jensen H: Visual dysfunctions and ocular disorders in children with developmental delay. I. prevalence, diagnoses and aetiology of visual impairment. Acta Ophthalmol Scand. 2007, 85:149-56. 10.1111/j.1600-0420.2006.00867.x

30. Kvarnström G, Jakobsson P, Lennerstrand G: Visual screening of Swedish children: an ophthalmological evaluation. Acta Ophthalmol Scand. 2001, 79:240-4. 10.1034/j.1600-0420.2001.790306.x 